\title{
A study of the role of calcium and oxidative stress in pathophysiology of osteoporosis in postmenopausal women-a review
}

\author{
Nour El-Houda Haddig, Aicha Zerzour, \& Samir Derouiche* \\ Department of Cellular and Molecular Biology, Faculty of Natural Sciences and Life, University of El Oued, El-Oued 39000, Algeria
}

\begin{abstract}
The aim of this review was to identify the role of calcium and oxidative stress as factors associated with osteoporosis in postmenopausal women. There are many diseases related to post-menstruation in women, the most important of which is osteoporosis. Calcium levels remains stable until menopause, when the bone resorption rate increases in association with the decrease in ovarian estrogen production that effect the intestinal calcium absorption. On the other hand, studies support that oxidative stress is directly involved in the genesis and development of osteoporosis. However, Oxidative stress blocks the activation of osteoblasts and activates the differentiation of osteoclasts which led to increased resorption rate without adequate and proper bone formation. In conclusion, Physiological changes in postmenopausal women lead to fluctuations in calcium metabolism and oxidative stress, which may lead to the occurrence or development of osteoporosis.
\end{abstract}

(C) 2020 Author(s). All rights reserved.

Keywords: Osteoporosis; Calcium; Oxidative stress; Menopause.

\section{Introduction}

Menopause (as defined by the World Health Organization) is the permanent cessation of menstruation due to loss of ovarian follicular activity (World Health Organization, 1981). And as the last menstrual period followed by at least twelve months of amenorrhea (no menstrual bleeding) (Shailendra, 2011). Usually occurs between the ages of 45 and 55 years (Southern, 2019). Menopause was an important selective trait in human evolution, as women who became infertile many years before death would be better able to care for existing children (Takahashi et al., 2017). In the other hand, calcium is an essential element; therefore, the bioavailability in the organism is generally insured by the food stuff (Kass-Wolff, 2004). Calcium status depends on the state of calcium metabolism, which is regulated by mechanisms including hormonal homeostasis, intestinal absorption, renal reabsorption and bone turnover. The hormonal part of regulation mechanism is based on parathyroid hormone (PTH), dihydroxyvitamin D, ionized calcium itself, and their corresponding receptors in the gut, kidney and bone (Derouiche and Kechrid, 2018). Calcium levels remains stable until menopause, when the bone resorption rate increases in association with the decrease in ovarian estrogen production that effect the intestinal calcium absorption (North American Menopause Society, 2006). Osteoporosis is a disease that is characterized by low bone mass, deterioration of bone tissue, and disruption of bone microarchitecture: it can lead to compromised bone strength and an increase in the risk of fractures (Zerzour et al., 2020). Osteoporosis, a multifactorial systemic skeletal disease, is characterized by low bone mineral density (BMD) and micro-architectural deterioration of bone tissue resulting in bone fragility. osteoporosis is the most prevalent disease in menopausal women, and is strongly associated with low quality of life ( $\mathrm{Ji}$ and $\mathrm{Yu}, 2015)$. Many of recent landmarks in scientific research have shown that in human beings, Oxidative stress is an important factor causing

\footnotetext{
* Corresponding author.

E-mail address: dersamebio@gmail.com (Samir Derouiche)
} 
metabolic and physiological alterations and various diseases in the body (Kaouachi and Derouiche, 2018), it is as a consequence of increase a reactive oxygen species and decrease in antioxidant defenses in prevalent in many health problems (Derouiche et al., 2019). In light of these data, the aim of this review was to identify the role of calcium and oxidative stress as factors associated with osteoporosis in postmenopausal women.

\section{Calcium and Risk factors of Osteoporosis}

In 2001, the National Institutes of Health has defined osteoporosis as "a skeletal disorder characterized by compromised bone strength predisposing a person to an increased risk of fracture (Gullberg et al.,1997). Osteoporosis is generally a disease of older adults because the cumulative effects of slow bone mineral loss take time to deplete the skeleton (Power et al., 2000). Physical activity/inactivity show good evidence that, among both women and men, physical inactivity is a risk factor for osteoporosis as well as for fractures (Cummings, 1955). Previous fractures are a major risk factor for new fractures (Kanis, 2004) And low weight is a higher risk for both osteoporosis and fractures (Brot et al., 1977). Also smoking decreases bone density and increases the risk of fractures (Forsen, 1994). And low exposure to sunlight leads to poor uptake of vitamin D (Johnell and Hertzman, 2006). Treatments with cortisone reduce bone density and increase the risk of fractures (Espallargues, 2001). Calcium is essential in maintaining total body health (Derouiche and Kechrid, 2013). When blood Ca levels drop too low, it is borrowed from the bones and returned to the bones from Ca supplied through the diet (Pravina et al., 2013). Thus, the effects of calcium deficiency may escape notice for a considerable time, until they manifest as skeletal weakness or fractures (Fischer et al., 2018). In fact, low dietary calcium intake is associated with low bone density, and calcium deficiency cause age-related bone loss, therefore, osteoporosis (Kim, 2014).

\section{Pathophysiology of Osteoporosis}

Once the peak bone mass is achieved, bone regulation takes place by local remodeling which is regulated by RANK (or receptor activator of nuclear factor-kb), RANKL (RANK Ligand) and OPG (Osteoprotegerin) (Kearns et al., 2008). RANK is present in the osteoclasts and causes the increase in their activity. RANKL is synthetized by osteoblasts and it binds with the RANK receptor on the osteoclasts. OPG is also synthetized by the osteoblasts and prevents the binding of the RANK to the RANK Ligand by itself binding to the RANKL (Wu et al., 2020). Hence, the activity of the osteoclasts and in turn the bone remodeling depends upon the interplay between the RANK and the OPG. This interplay is controlled by hormonal and local factors (Vikram et al., 2017). Imbalance in bone remodeling lead to decreased skeletal mass. In most individuals, bone mass peaks in the third decade, after which bone resorption exceeds bone formation. Failure to reach a normal peak bone mass or acceleration of bone loss can lead to osteoporosis (Varacallo and Fox, 2014).

\section{Osteoporosis in menopausal women}

A decline in estrogen has been shown to play a major role in the decreasing bone mass during the menopause, especially because it has a variety of protective effects on bone marrow and bone cells (Manolagas et al., 2013). This hormone allows for increased bone formation by reducing the production and function of the osteoclasts as well as increasing osteoclast apoptosis (Jilka et al., 1999). This effect on the osteoclastic cells of the bone is facilitated via estrogen's inhibition of a signaling molecule, RANKL, which is involved in osteoclast differentiation and survival. However, due to the estrogen deficiency during menopause, this beneficial effect on the bone is lost causing osteoporosis (Doshi and Agarwal 2013). Also, menopause has been linked to an increase in inflammatory cytokines within the serum specifically tumor necrosis factor (TNF- $\alpha$ ), which negatively impacts the bone by contributing to increased osteoclast formation. Additionally, the high levels of FSH during menopause stimulate osteoclast differentiation and TNF- $\alpha$ production, both of which play an important role in osteoporotic bone loss (Graziana et al., 2013). 


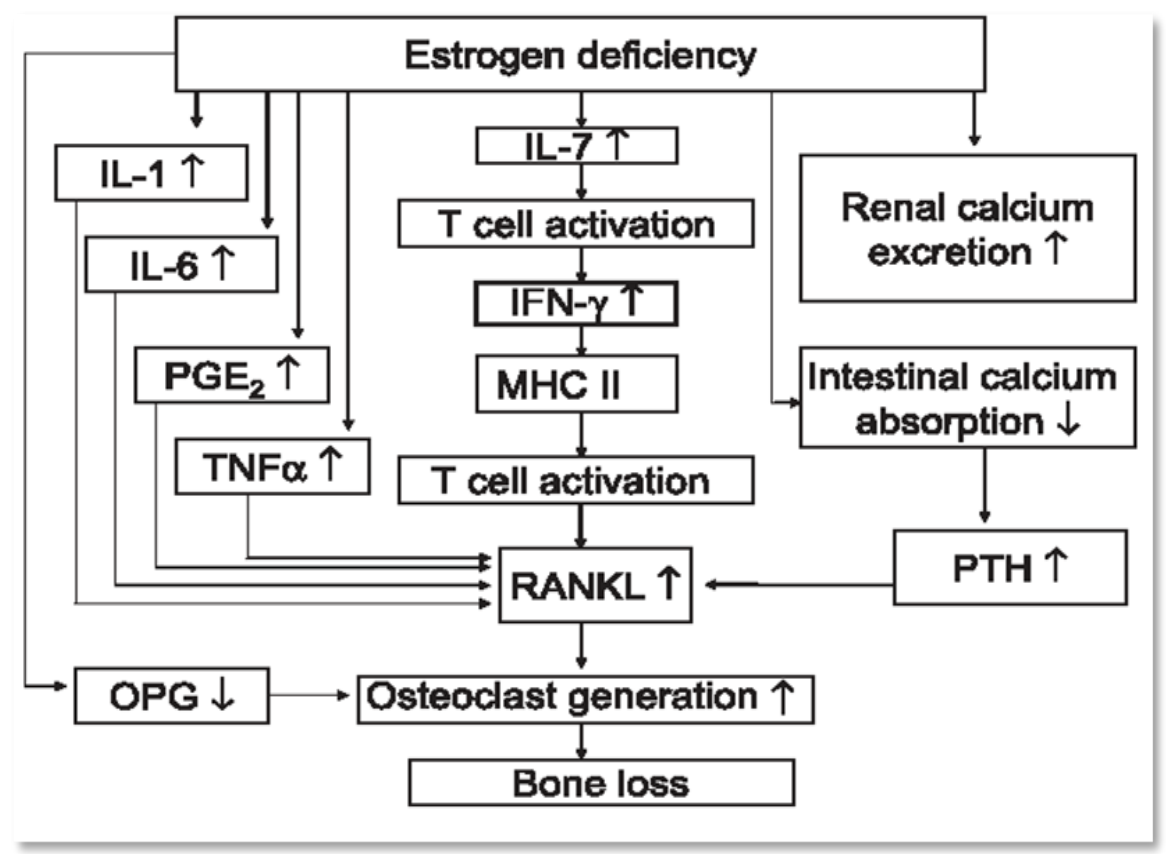

Fig. 1. A model of the effects of estrogen deficiency on bone loss, prostaglandin E2 and Osteoprotegerin (Wolfgang et al., 2009)

\section{Oxidative stress and osteoporosis}

Oxidative stress is defined as an imbalance in the balance between antioxidants and pro-oxidants in favor of antioxidants (Derouiche et al., 2017; Boulaares et al., 2020). The overproduction of free radicals can cause oxidative damage to biomolecules (lipids, proteins, DNA), eventually leading to many chronic diseases such as cancer, diabetics, cardiovascular diseases, chronic inflammation, aging and osteoporosis (Derouiche et al., 2018).

\subsection{Oxidative stress during menopause}

Menopause creates a redox imbalance and subsequent oxidative stress due to the decline of the natural antioxidant hormone estrogen (Sankar et al., 2017). Specifically, at high concentrations, estrogen tends to have a beneficial antioxidant effect by inhibiting the 8-hydroxylation of guanine DNA bases (Doshi and Agarwal, 2013), also by increasing the activities of antioxidant enzymes, such as glutathione peroxidase, and causing an increase in antioxidant vitamin levels (Atoussi et al., 2018; chetehouna et al., 2020). However, an increasing number of studies have recently shown another activity of estrogens as important pro-oxidants at physiological concentrations (Victorino et al., 2013) and changes in the lipid profile and the increase of lipoperoxidation (Montoya-Estrada et al., 2020). Additionally, serum concentrations of inflammatory cytokines and pro-oxidant biomarkers such as glutathione, 4-hydroxynenal, and malonaldehyde were found to be higher in postmenopausal women than in premenopausal women (Agarwal et al., 2005). The elevation of cytokines and pro-oxidant makers suggests that there is a high degree of oxidative stress in the postmenopausal state (Derouiche, 2020).

\subsection{Oxidative stress and osteoporosis}

Oxidative stress activates the differentiation of osteoclasts which led to a significant increase in the number and activity of these cells. Also, ROS induce the apoptosis of osteoblasts and osteocytes, by activating numerous signaling 
pathways (Domazetovic et al., 2017). The oxidative stress blocks the activation of osteoblasts and thus the production of OPG; under this condition, the action of RANKL prevails, and the differentiation and activity of osteoclasts are induced. Thereafter, the turnover of the bone remodeling process increases due to increased resorption rate without adequate and proper bone formation; this event has been related to osteoporosis (Domazetovic et al., 2017). Antioxidants have opposing effects, they contribute to the differentiation of osteoblasts and bone formation, while reduce the osteoclast differentiation and their activity (Agidigbi and Kim, 2019).

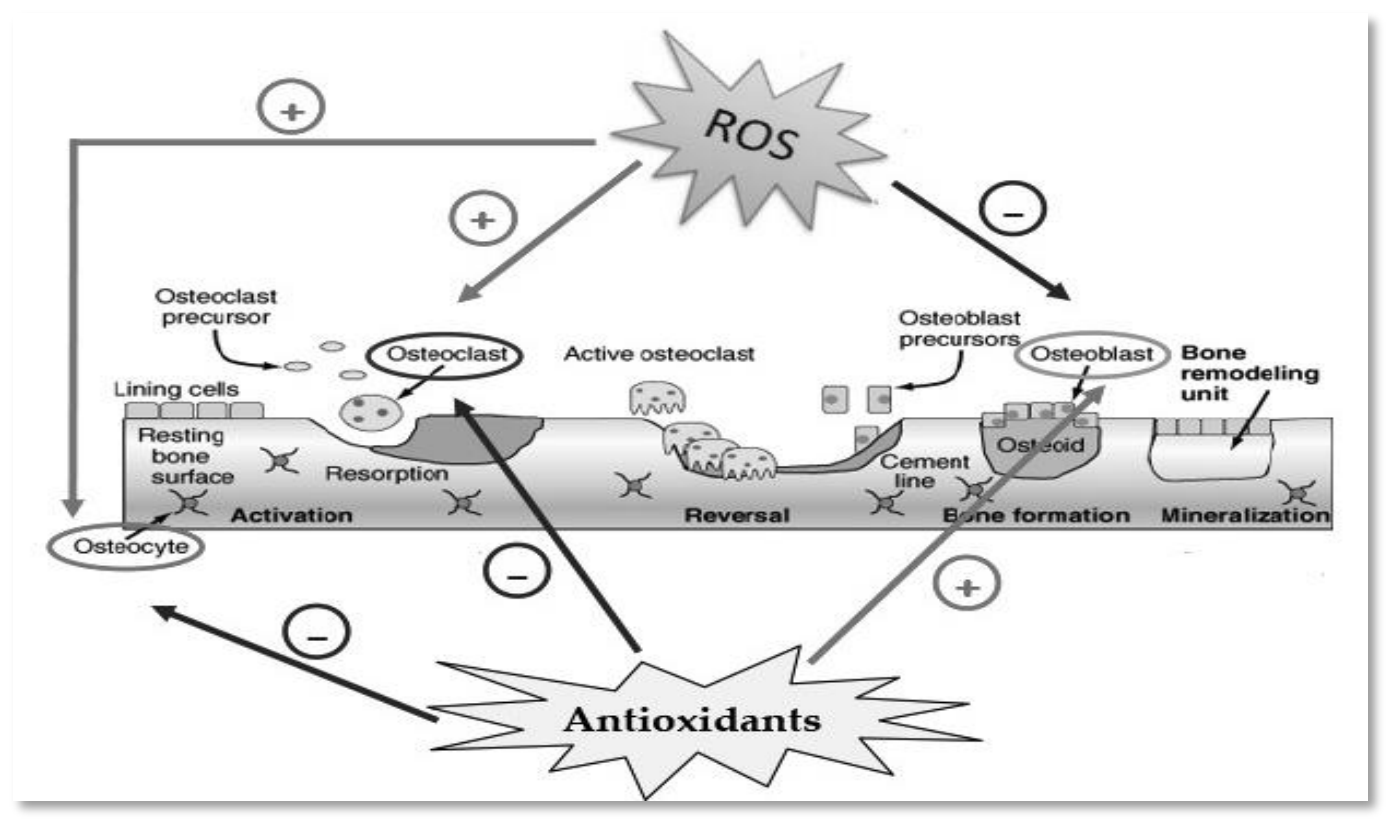

Fig. 2. Effect of ROS and antioxidants on the activity of bone cells in bone remodelling (Domazetovic et al., 2017).

Reactive oxygen species (ROS) are free radicals resulting from the metabolism of oxygen (Derouiche et al., 2020) which activate osteoclast differentiation and osteocyte apoptosis (+), while inhibit osteoblast activity (-) inducing bone resorption; antioxidants activate osteoblast differentiation $(+)$ and inhibit osteoclast activity and osteocyte apoptosis (-) inducing bone formation (figure) (Almeida, 2012).

\section{Conclusion}

Physiological changes in postmenopausal women lead to fluctuations in calcium metabolism and oxidative stress, which may lead to the occurrence or development of osteoporosis. On the other hand, oxidative stress is essential factor in the development and complication of the osteoporosis and therefore it is necessary to take into account the mitigation of these phenomena in any approved treatment program which contributes to the prevention or limitation of disease development.

\section{References}

Agarwal, A., Nallella, K.P., Allamaneni, S.S., et al. (2005). Role of oxidative stress in female reproduction. Reproductive Biology and Endocrinology, 3,28. 
Agidigbi, T.S. \& Kim, C. (2019). Reactive Oxygen Species in Osteoclast Differentiation and Possible Pharmaceutical Targets of ROS-Mediated Osteoclast Diseases. International Journal of Molecular Sciences, 20(14),3576.

Almeida, M. (2012). Aging mechanisms in bone. BoneKEy Reports, 1, 102.

Atoussi, N., Guediri, S., Derouiche, S. (2018). Changes in Haematological, Biochemical and Serum Electrolytes Markers in Women Breast Cancer Patients. Scholars Journal of Research in Agriculture and Biology, 3(2), 73-177.

Boulaares, I., Guemari, I.Y., Derouiche S. (2020). Analysis of Some Hematological and Biochemical Markers in Women Cancer Patients Receiving Doxorubicine Chemotherapy. Pharmaceutical and Biosciences Journal, $8(1), 29-34$.

Brot, C., Jensen, L. \& Sorensen, O. (1977). Bone mass and risk factors for bone loss in perimenopausal Danish women. Internal Medicine. 242(6), 505-511.

Chetehouna, S., Atoussi, O. \& Derouiche S. (2020) Biological Activity and Toxicological Profile of Zinc Oxide Nanoparticles Synthesized by Portulaca oleracea (L) Leaves Extract. Advance in Nanomedicine and Nanotechnology Research, 2(2), 125-133.

Cummings, S. (1955). Risk factors for hip fracture in white women. New England Journal of Medicine. 332(12), 767773.

Derouiche S, Abbas K, Djermoune M. (2017). Polysaccharides and ascorbic acid content and the effect of aqueous extract of portulaca oleracea in high-fat diet-induced obesity, dyslipidemia and liver damage in albino wistar rats. Algerian Journal of Arid Environment. 7(2): 16-26.

Derouiche, S. \& Kechrid, Z. (2013). Influence of calcium supplements on zinc status, carbohydrate metabolism and the liver activity of detoxifying glutathione enzymatic system in alloxan induced diabetic rats. Journal Of Experimental Biology and Agriculture Sciences, 1(6), 424-429.

Derouiche, S. (2020). Oxidative Stress Associated with SARS-Cov-2 (COVID-19) Increases the Severity of the Lung Disease - A Systematic Review. Journal of Infectious Diseases and Epidemiology, 6,121.

Derouiche, S., \& Kechrid Z. (2018). Zinc Supplementation Attenuated Calcium-High Diet Effect on Zinc Status and Carbohydrate Metabolism of Non-Diabetic and Diabetic Rats. International Journal of Diabetes and Clinical Research, 5(4): 095.

Derouiche, S., Cheradid, T., Abdelmolk, D. \& Achi, I. (2020). Effect of COVID-19 Infection on the Immune System and Risk of Developing Diabetes Complications: A Review. Journal of Pharmacy Care. 8(3), 133-139.

Derouiche, S., Rezzag-mohcen, O.S. \& Serouti, A. (2018). Triazinone herbicide metribuzin induced acute liver injury: A study of animal model. Journal of Acute Diseases, 7(4), 152-157.

Derouiche, S., Serouti, A., \& Rezzag-mohcen, O.S. (2019). Risk of Metribuzin (Triazinone herbicide) on hematological and renal structure and function of pregnancy rabbits. Asian Journal of Biological Sciences, 12,192-198.

Domazetovic, V., Marcucci, G., Iantomasi, T., Brandi, M.L. \& Vincenzini, M.T. (2017). Oxidative stress in bone remodeling: role of antioxidants. Clinical Cases in Mineral and Bone Metabolism, 14(2), 209-216.

Doshi, S.B. \& Agarwal, A. (2013). The role of oxidative stress in menopause. Mid-life Health, 4(3), 140-146.

Espallargues, M. (2001). Identifying bone-mass-related risk factors for fracture to guide bone densitometry measurements: a systematic review of the literature. Osteoporosis International, 12(10), 811-822. 
Fischer, V., Haffner-Luntzer, M., Amling, M. \& Ignatius, A. (2018). Calcium and vitamin d in bone fracture healing and post-traumatic bone turnover. European Cells and Materials, 35, 365-385.

Forsen, L. (1994). Interaction between current smoking, leanness, and physical activity in the prediction of hip fracture. Bone and Mineral Research, 9(11), 1671-1678.

Graziana, C., Concetta, C. \& Silvia, C. (2013). FSH and TSH in the Regulation of Bone Mass: The Pituitary/Immune/Bone Axis. Journal of Immunology research. Article ID 382698.

Gullberg, B., Johnell, O. \& Kanis, J.A. (1997). World - wide projections for hip fracture. Osteoporosis international, 7(5): 407-413.

Ji, M.X. \&Yu, Q. (2015). Primary osteoporosis in postmenopausal women. Chronic Diseases and Translational Medicine, 1(1), 9-13.

Jilka, R.L., Weinstein, R.S., Bellido, T., Roberson, P., Parfitt, A.M., Manolagas, S.C. (1999). Increased bone formation by prevention of osteoblast apoptosis with parathyroid hormone. Journal of Clinical Investigation, 104(4),439-46.

Johnell, O. \& Hertzman, P. (2006). What evidence is there for the prevention and screening of osteoporosis?. Health Evidence Network report. Copenhagen: World Health Organization.

Kanis, J. (2004). A meta-analysis of previous fracture and subsequent fracture risk. Bone, 35(2), 375-382.

Kaouachi, A. \& Derouiche, S. (2018). Phytochemical analysis, DPPH antioxidant activity and Acute toxicity of bark aqueous extracts of Pinus halepensis. Research Journal of Chemical and Environmental Sciences, 6(3), 86-91.

Kass-Wolff JH. (2004). Calcium in women: Healthy bones and much more. Journal of Obstetrics Gynecology and Neonatal Nursing, 33, 21-33.

Kearns, A.E., Khosla, S. \& Kostenuik, P.J. (2008). Receptor activator of nuclear factor kappa B ligand and osteoprotegerin regulation of bone remodeling in health and disease. Endocrine Reviews, 29(2),155-92.

Kim, M.K., Hee-Choi, S., Lim, S., Moon, J.H., Hee-Kim, J., Wan-Kim, S., Chul-Jang, H. \& Soo-Shin, C. (2014). Interactions Between Dietary Calcium Intake and Bone Mineral Density or Bone Geometry in a Low Calcium Intake Population (KNHANES IV 2008-2010). Clinical Endocrinology \& Metabolism, 99(7), 2409-2417.

Manolagas, S.C., Charles, A.O., et al. (2013). The role of estrogen and androgen receptors in bone health nd disease. Nature Reviews Endocrinology, 9(12), 699-712.

Montoya-Estrada, A., Velázquez-Yescas, K.G., Veruete-Bedolla, D.B., Ruiz-Herrera, J.D., Villarreal-Barranca, A., Romo-Yañez, J., Ortiz-Luna, G.F., Arellano-Eguiluz, A., Solis-Paredes, M., Flores-Pliego, A., Espejel-Nuñez, A., Estrada-Gutierrez, G., Reyes-Muñoz, E. (2020). Parameters of Oxidative Stress in Reproductive and Postmenopausal Mexican Women. Environmental Research and Public Health, 17(5), 1492.

North American Menopause Society. (2006). Management of osteoporosis in postmenopausal women: 2006 position statement of The North American Menopause Society. Menopause, 13(3), 340-367.

Power, M.L., Heaney, R.P., Kalkwarf, H.J., Pitkin, R.M., Repke, J.T., Tsang, R.C., Schulkin, J. (2000). The role of calcium in health and disease. Obstetrics and Gynecology, 181(6), 1560-1569.

Pravina, P., Sayaji, D. \& Avinash, M. (2013). Calcium and its Role in Human Body. International Journal of Research in Pharmaceutical and Biomedical Sciences, 4(2), 660-661.

Sankar, P., Bobby, Z. \& Mirza, A.A. (2017). Soy Isoflavones (from Glycine max) in Menopause Health and Diseases. Biochemistry \& Physiology, 6(3), 2180-2192.

Shailendra K. (2011). Menopausal transition and postmenopausal health problems: a review on its biocultural perspectives. Health, 3(4), 233-237.

Southern, C. (2019). Menopause: Signs, symptoms and treatment. Medical Library Index. 
Takahashi, M., Singh, R.S., Stone, J. (2017). A Theory for the Origin of Human Menopause. Front Genet., 7, 222.

Varacallo, M.A. \&Fox, E.J. (2014). Osteoporosis and its complications. Medical Clinics of North America, 98(4), 817-831.

Victorino, V.J., et al. (2013). Decreased oxidant profile and increased antioxidant capacity in naturally postmenopausal women. Age (Dordr), 35(4),1411-21.

Vikram, K., Parul, G., Ranjana, K. \& Harish, C. (2017). Postmenopausal Osteoporosis. Department of Orthopaedics, Ranjana Hospital, Allahabad, India. Juniper Online Journal of Case Studies, 1(4), 1-5.

Wolfgang, S., Peter, P. \& Janina, P. (2009). Pathophysiology of osteoporosis. Wiener Medizinische Wochenschrift, 159(9), 230-234.

World Health Organization. (1981). Research on the menopause: WHO technical report series (No.670.). Geneva: World Health Organization.

Wu, X., Li, F., Dang, L., Liang, C., Lu, A. \& Zhang, G. (2020). RANKL/RANK System-Based Mechanism for Breast Cancer Bone Metastasis and Related Therapeutic Strategies. Frontiers in Cell and Developmental Biology, 8,76 .

Zerzour, A., Heddig N.E., \& Derouiche S. (2020). Analysis of Osteoporosis risk factors in Menopausal women's of Algeria population. Asian Journal of Research and Pharmeutical. Sciences, 10(2),79-84. 